This item was submitted to Loughborough's Research Repository by the author.

Items in Figshare are protected by copyright, with all rights reserved, unless otherwise indicated.

\title{
Particle-by-Particle Charge Analysis of DNA-Modified Nanoparticles Using Tunable Resistive Pulse Sensing
}

PLEASE CITE THE PUBLISHED VERSION

http://dx.doi.org/10.1021/acs.langmuir.5b03024

\section{PUBLISHER}

(C) American Chemical Society

\section{VERSION}

AM (Accepted Manuscript)

\section{PUBLISHER STATEMENT}

This work is made available according to the conditions of the Creative Commons Attribution-NonCommercialNoDerivatives 4.0 International (CC BY-NC-ND 4.0) licence. Full details of this licence are available at: https://creativecommons.org/licenses/by-nc-nd/4.0/

\section{LICENCE}

CC BY-NC-ND 4.0

\section{REPOSITORY RECORD}

Blundell, Emma L.C.J., Robert Vogel, and Mark Platt. 2019. "Particle-by-particle Charge Analysis of Dnamodified Nanoparticles Using Tunable Resistive Pulse Sensing". figshare. https://hdl.handle.net/2134/20179. 


\title{
Particle-by-Particle Charge Analysis of DNA-Modified
} Nanoparticles Using Tunable Resistive Pulse Sensing

\author{
Emma L. C. J. Blundell ${ }^{1}$, Robert Vogel ${ }^{2,3}$ and Mark Platt ${ }^{{ }^{*}}$
}

${ }^{1}$ Department of Chemistry, School of Science, Loughborough University, Loughborough, Leicestershire, LE11 3TU, United Kingdom

${ }^{2}$ Izon Sciences Limited, 8C Homersham Place, PO Box 39168, Burnside, Christchurch 8053, New Zealand

${ }^{3}$ School of Mathematics and Physics, The University of Queensland, Brisbane 4072, Australia

*Correspondence should be sent to m.platt@lboro.ac.uk, Fax +44(0)1509 223925 


\begin{abstract}
Resistive pulse sensors, RPS, are allowing the transport mechanism of molecules, proteins and even nanoparticles to be characterised as they traverse pores. Previous work using RPS has shown that the size, concentration and zeta potential of the analyte can be measured. Here we use tunable resistive pulse sensing (TRPS) which utilises a tunable pore to monitor the translocation times of nanoparticles with DNA modified surfaces. We start by demonstrating that the translocation times of particles can be used to infer the zeta potential of known standards and then apply the method to measure the change in zeta potential of DNA modified particles. By measuring the translocation times of DNA modified nanoparticles as a function of packing density, length, structure, and hybridisation time, we observe a clear difference in zeta potential using both mean values, and population distributions as a function of the DNA structure. We demonstrate the ability to resolve the signals for ssDNA, dsDNA, small changes in base length for nucleotides between 15-40 bases long and even the discrimination between partial and fully complementary target sequences. Such a method has potential and applications in sensors for the monitoring of nanoparticles in both medical and environmental samples.
\end{abstract}

Key words: Biosensor; TRPS; Zeta potential; DNA; Superparamagnetic particles. 


\section{Introduction}

The immobilization of oligonucleotides onto surfaces is a key design to many technologies within DNA sequencing ${ }^{1,2}$, DNA-protein interactions ${ }^{3-5}$, biosensing ${ }^{6-9}$ and targeted drug delivery ${ }^{10-12}$. The functionalization of DNA onto nanoparticle surfaces is now a common practice, and within the field of biosensors alone the number of strategies for immobilization, type of nanomaterial, and detection platform are varied enough to fill several reviews ${ }^{13-19}$. One family of nanomaterials favoured with purification strategies is Superparamagnetic Particles, SPPs. These particles allow for the removal of specific analytes from complex sample matrices using nothing more complicated than a hand held magnet ${ }^{14,20-23}$ and the use of SPPs has become increasingly common. When they are incorporated into fluidic devices they can be used to continuously sort cells and DNA from liquids ${ }^{24}$, and are integrated into a variety of detection platforms ${ }^{14,24,25}$.

When using nanomaterials in bioassays, the material must remain suspended in the solution for it to capture the analyte. A particle's surface chemistry design is important to avoid sedimentation of irreversible aggregation; there are two mechanisms available to prevent this. First is the use of steric stabilisation by placing a neutral polymer onto the particle surface, and the second depends upon charge stabilisation whereby the repulsive coulombic forces overcome the attractive Van der Waals forces ${ }^{26,27}$.

For charge stabilised particles, a typical measurement used to represent the surface charge, and infer stability, is zeta potential. The zeta potential represents the value of the electrostatic potential at the plane of shear and typically for nanoparticle systems, zeta potential values of $\pm 30 \mathrm{mV}$ are representative of stabilised particles ${ }^{28}$. When a polyelectrolyte, such as DNA, is immobilised onto the surface of the nanomaterials the DNA can take on two roles. The first is the more natural of the two as a capture probe, designed to hybridise to target DNA. The second is a passive role where the inherent charge on the phosphate back bone can act as a stabiliser by creating a high charge density on the particle surfaces, helping suspend them in solution $^{29}$. In doing this it is important to consider the structure of the DNA immobilised onto a nanomaterial's surface. Single-stranded and double-stranded 
DNA varies in persistence length, which affects the stability and flexibility of the polymer when immobilised to a surface. For example, dsDNA has a 50-fold higher persistence length than $s s D N A^{30,31}$, making it a far more rigid. As well as the persistence length, the contour length also varies between ssDNA and dsDNA, and both these parameters will affect the plane of shear and thus zeta potential ${ }^{32}$.

When using nanoparticle systems a mean population zeta potential will not allow the true measure of the ligand distribution across all of the particles to be interpreted, and in a typical reaction the ligand density would follow a poisson distribution $^{33-35}$. The spread of the population can have an effect on the reaction kinetics, stability and sensitivity of nanoparticle based assays ${ }^{36-38}$. To build up a true measure of the spread of zeta potential values for a given particle population, the zeta potential of each individual particle has to be measured, and this aspect is challenging, although electrophoretic and electrochemical techniques allow insight to these measurements ${ }^{29,39}$. Electrophoresis studies have demonstrated the ability to separate ssDNA and dsDNA modified particles, and probe the structure of the ssDNA surfaces ${ }^{40-42}$. Alternative technologies for monitoring particle-by-particle zeta potentials rely upon particle tracking technologies that monitor the speed of the particles in an applied electric field ${ }^{43}$.

A relatively recent technology to be developed for the characterisation of nanoparticles is based upon tunable resistive pulse sensing (TRPS) ${ }^{44-51}$. TRPS is based on polyurethane elastomeric membranes in which the pore geometry can be altered in real time. The brief set up and theory for TRPS technologies is as follows: a stable ionic current is established by two electrodes, separated by a pore; as particles/analytes translocate the pore they temporarily occlude ions, leading to a transient decrease in current known as a "blockade event", examples of which can be seen in figure 1a. In the TRPS arrangement used here, the pore is mounted laterally so that particles typically move from the upper fluid cell into the lower fluid cell, aided by an inherent pressure head due to $40 \mu \mathrm{l}$ of liquid in the upper fluid cell of approximately $50 \mathrm{~Pa}^{52}$, and a positive or negative bias is applied via an electrode under the pore. By monitoring changes in blockade width or full width half maximum (FWHM), blockade magnitude $\left(\Delta \mathrm{i}_{\mathrm{p}}\right)$ and blockade frequency (events/min) it is possible to elucidate the zeta potential ${ }^{53,54}$, size $e^{49}$, and concentration ${ }^{50}$ of colloidal dispersions in situ ${ }^{49}$. 
a)

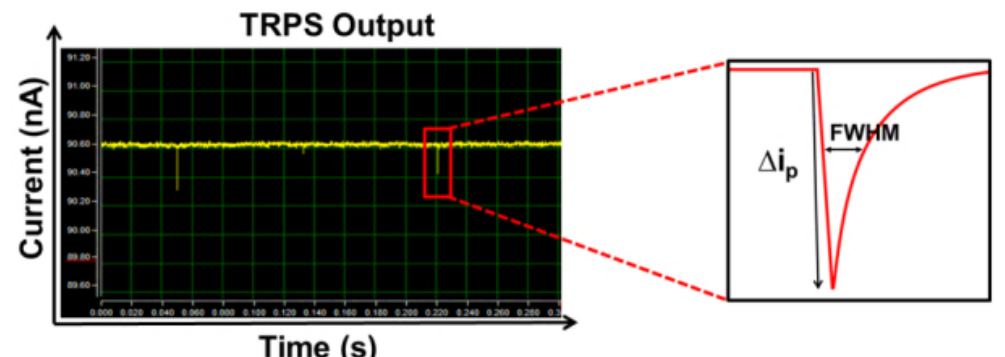

b)

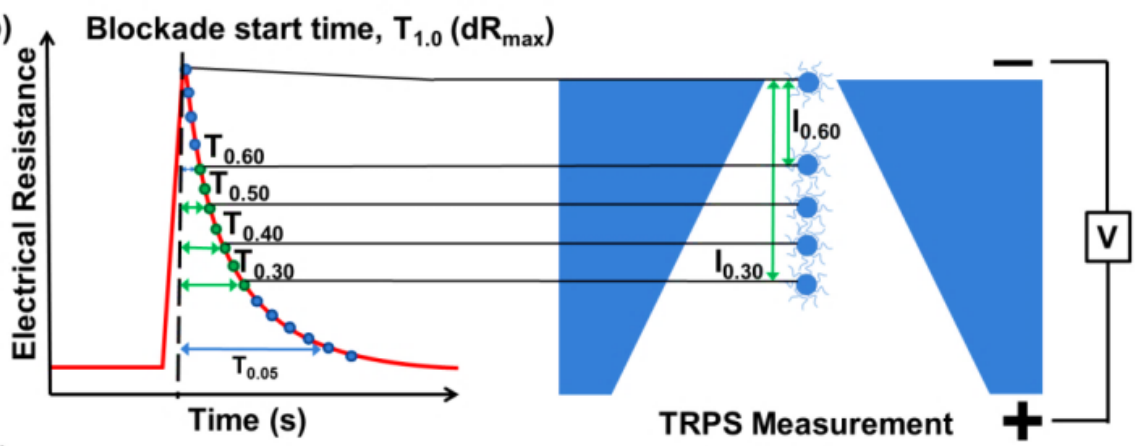

c)

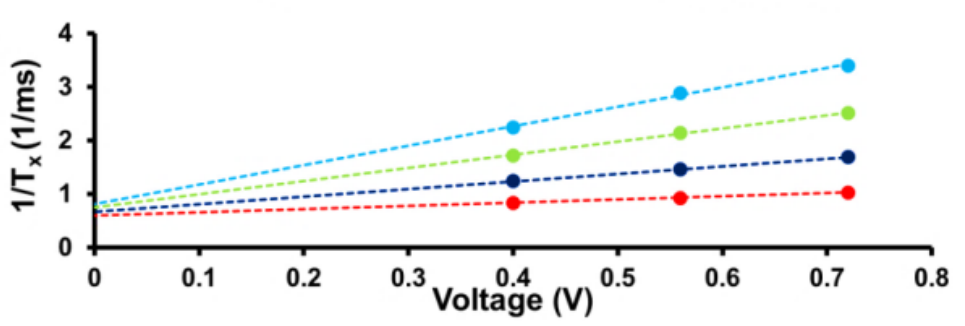

Figure $\mathbf{1}$ - a) Blockade events produced as particles traverse the pore and the resulting blockade shape depicting $\triangle i_{p}$ and FWHM. b) Blockade shape is relative to particle translocation and a measurement is made at 8 blockade reference points representing a particle's position in the nanopore at any given time (green spots). $T_{1.0}$ for example is the time the blockade is at $100 \%$ magnitude, $d R_{\max } . T_{0.30}$ and $T_{0.60}$ are the times at which the blockade is $30 \%\left(I_{0.30}\right)$ and $60 \%\left(I_{0.60}\right)$ of $d R_{\max }$ respectively. c) $1 / T$ vs voltage used within the calibration method to calculate the particle translocation time and therefore zeta potential of a given sample.

The methodology for measuring zeta potential using RPS technologies has seen an evolution of techniques ${ }^{44,54,55}$, and here we use a similar concept as was published by Arjmandi et. al. using pyramidal pores ${ }^{56}$. In brief, a calibration based zeta potential method is applied, based on the measurement of signal durations of translocation events as a function of voltage. The electrophoretic mobility is calculated from the derivative of medium particle velocity and applied electric field. The zeta potential of each particle can then be obtained from the measured electrophoretic mobility using the Smoluchowski approximation ${ }^{44,57}$. The calculated 
zeta potential only depends on the measured pulse duration and is independent of the magnitude of the pulse, meaning that simultaneous size and charge measurements can easily and reliably be carried out. TRPS's particle-by-particle nature means that sub-populations with different zeta potential are able to be resolved, whilst ensemble methods such as phase analysis light scattering or PALS will only report an average zeta potential.

We adapt and apply the theory by first demonstrating its use with calibration particles and then move to measure changes in zeta potential for DNA modified nanoparticles. We go on to measure the change in zeta potential as a function of DNA concentration on the particles surface. We observe that the measured zeta potential is correlated to the concentration of DNA and as the technique also provides a particle-by-particle analysis, the distribution of the zeta potential across the sample population is also produced. As we increase the concentration of DNA, a more symmetrical Gaussian distribution of charge is produced, indicating a more uniform ligand distribution around the nanoparticles. By measuring the zeta potential and shape of the distribution, we go on to measure the effects of oligonucleotide length and apply our method to the detection of dsDNA. By controlling the packing density of the capture probe (CP) on the particle surface and the mechanism by which the CP hybridises to the target, the sensitivity of the instrument can allow for the detection of target DNA in assay times under 30 mins. Finally we demonstrate that by designing the length and position of the complementary section to the target we can improve the signal and detection.

The method will have an impact on designing particle based assays and the technology shows potential to study zeta potentials on biological analytes, with clear applications in fields of bioassays; as well as the monitoring of nanomaterials in nanotoxicology and nanomedicine where a clear understanding of the particle surface charge and size can have an influence on the efficiency and toxicology of particle based drugs.

\section{Materials and methods}

\subsection{Chemicals and Reagents}


The buffer used was phosphate buffered saline with Tween-20 as a surfactant (1 x PBST (0.01 M phosphate buffer, 0.0027 M Potassium Chloride, 0.137 M Sodium Chloride, pH 7.4 with $0.05(\mathrm{v} / \mathrm{v}) \%$ Tween-20 in $200 \mathrm{~mL}$ deionised water $(18.2 \Omega \mathrm{cm}))$ ). PBS tablets (P4417) and Tween-20 (P1379) were purchased from Sigma Aldrich, UK. Streptavidin coated superparamagnetic particles $(120 \mathrm{~nm}, 4352 \mathrm{pmol} / \mathrm{mg}$ binding capacity, product 03121) were purchased from Ademtech, France.

\subsection{Carboxyl polystyrene particle standards}

Carboxylated polystyrene particles with a mean nominal diameter of $220 \mathrm{~nm}$ were purchased from Bangs Laboratories, US and are denoted as CPC200. The specific surface charge as determined by the manufacturer was $86 \mu \mathrm{eq} / \mathrm{g}$, equivalent to a surface density of $3.2 \times 10^{-19} \mathrm{C} / \mathrm{nm}^{2}$. The CPC200s were measured at a concentration of $1 \times 10^{10}$ particles $/ \mathrm{mL}$.

\subsection{Custom DNA oligonucleotides}

All the oligonucleotides used in this study were purchased as lyophilised powders (100 pmol/ $\mu \mathrm{L})$ from Sigma Aldrich, UK with customised DNA sequences fit for purpose detailed below, please not the abbreviation [Btn] is relative to a biotin modification; 5'NNNNNNNNNN[Btn]3' (VL10, 10 bases), 5'TGGGAGTAGGTTGGTGTGGTTGGGGCTCCCCTTTTT[Btn]3' (VL36, 36 bases), 5'ATACCAGTCTATTCAATTGGGCCCGTCCGTATGGTGGGTGTGCTGGCCAG[Btn ]3' (VL50, 50 bases), 5'ATGGTTAAACCTCACTACGCGTGGC[Btn]3' (VL25/CP, 25 bases), 5'GCCACGCGTAGTGAGGTTTAACCAT3' (cDNA, 25 bases), 5'GTAGTGAGGT3' (MidT, 10 bases), 5'GTTTAACCAT3' (EndT, 10 bases), 5'GTGAGGTTTAACCATTTTTTTTTTTTTTTT3' (OverT, 30 bases).

\subsection{Phase analysis light scattering (PALS)}

CPC200 zeta potentials were measured on a Malvern Zetasizer Nano ZS. PALS analysis was used to determine the average zeta potential of the carboxylated polystyrene standards dispersed in PBS electrolyte. 


\subsection{Hybridising DNA to streptavidin coated particles}

$120 \mathrm{~nm}$ diameter streptavidin coated particles (03121, Ademtech, France) were diluted to a concentration of approximately $1 \times 10^{9}$ particles $/ \mathrm{mL}$. The diluted particle solutions were then vortexed for $30 \mathrm{~s}$, and sonicated for 2 mins, to ensure monodispersity.

The biotinylated DNA capture probe was added to the streptavidin coated particles (4352 pmol/mg binding capacity - determined by the supplier) at the required concentration. The samples were then placed on a rotary wheel for 30 minutes. Any unbound DNA remaining in solution was then removed via magnetic separation by placing the samples onto a Magrack (GE Healthcare, UK) for 30 minutes. The supernatant was then removed and replaced with new buffer (PBST).

\subsection{Addition of complementary target DNA}

Target DNA was added in excess (500 nM) to ensure the maximum possible target binding was reached. The samples were then placed on a rotary wheel at room temperature to investigate the effect of DNA hybridisation time.

\subsection{TRPS setup}

All measurements were conducted using the qNano (Izon Sciences Ltd, NZ) combining tunable nanopores with proprietary data capture and analysis software, Izon Control Suite v.2.2.2.117. The lower fluid cell always contained the electrolyte buffer $(80 \mu \mathrm{L}$ ). The upper fluid cell always contained $40 \mu \mathrm{L}$ of sample (that was suspended in the buffer) when a measurement was being completed with an inherent pressure on the system (47 Pa). Prior to TRPS analysis, all samples were vortexed for $30 \mathrm{~s}$ and sonicated for 1 minute. During each sample run, the system was washed by placing PBST $(40 \mu \mathrm{L})$ into the upper fluid cell several times with various pressures applied to ensure there were no residual particles remaining and therefore no cross contamination between samples. A detailed description of such a tunable resistive pulse sensing device can be found in Willmott et al. ${ }^{50}$ and Vogel et al. ${ }^{49}$. 


\subsection{Streaming potential measurement}

Streaming potential and current measurements of the thermoplastic polyurethane (TPU) pore membrane were made using a Surpass instrument (Anton Paar GMBH, USA). TPU membranes were cut to form a cylindrical cell with an adjustable gap and the streaming potential was measured for a range of applied pressures within a cyclic pressure sweep. The zeta potential was evaluated with the Surpass Visiolab software, applying the Helmholtz-Smoluchowski equation ${ }^{58}$.

\subsection{Calibration zeta potential measurements}

Based on the size of the sample particles being used $(\sim 120 \mathrm{~nm})$ and calibration CPC200s $(220 \mathrm{~nm}$ ), the most idealistic pore to use was an NP150 (size range $85-300 \mathrm{~nm}$ ). The calibration particles are measured at 3 applied voltages that are dependent on the applied stretch and consequent baseline current observed. Each sample measurement was performed using a baseline current of $100 \pm 10 \mathrm{nA}$, to allow us to compare data sets across several runs and pores. To account for variation in the pore size from the manufacturing process, the stretch and voltage were adjusted to achieve a similar baseline current for each experiment (see above). As well as matching the baseline current each sample blockade signal was greater than $0.05 \mathrm{nA}$, compared to a background noise of circa $10 \mathrm{pA}$. Finally when performing an experiment a calibration was performed on particles of known size and zeta potential. For the purpose of measuring and comparing zeta potential, it was imperative that the stretch of the nanopore and the applied potential were not changed during a sample or calibration measurement of a particular dataset. The sample measurements were all completed at the highest or second highest voltage that the calibration measurements were carried out at. Calibration measurements were completed on each new day analysis was completed and when a new nanopore was introduced. The zeta potential distributions were measured as $\mathrm{D} 90 / 10$ value, $\mathrm{D} 90$ is defined as the particle zeta potential at $90 \%$ of the cumulative particle zeta potential, distribution and D10 is defined as the particle zeta potential at $10 \%$ of the cumulative particle zeta potential. 


\section{Results and discussion}

\subsection{Zeta calculation}

In this study we are using a method related to Arjmandi et al., ${ }^{56}$, who described a calibration based method of measuring particle zeta potentials using resistive pulse sensing. This method is based on measuring the duration of the translocation of particles through a nanopore as a function of applied voltage, with particle velocity and electric field being averaged over the entire sensing zone of a regular conical pore. The electric field, $E$, can be determined using the calculation of pore resistance, so that $E$ is entirely parallel to the $z$-axis, $E_{z}(z)=-I_{0} * \frac{d R}{d z}$, with $E_{z}, I_{0}$, and $R$ being the electric field component along the pore axis, electric current, and resistance respectively ${ }^{59}$. For a voltage $V_{0}$ of $0.5 \mathrm{~V}$, a small pore opening diameter of $0.8 \mu \mathrm{m}$, a large pore opening diameter of $40 \mu \mathrm{m}$, and a membrane thickness of $250 \mu \mathrm{m}$, the maximum electric field is approximately $10^{5} \mathrm{~V} / \mathrm{m}$. Please note that the above pore dimensions are estimates, which are in accordance with SEM images of pores with similar dimensions to the ones used for this study. The electrophoretic mobility is the derivative of $1 / T$ (with $T$ being the signal duration) and voltage multiplied by the square of the sensing zone length, I. I is a fitting parameter that is included in a calibration constant, which is calculated using a calibration particle with known zeta potential. Convection and electroosmosis have been neglected for being much smaller than the electrophoretic contribution to particle motion. Finally, Henry's equation is used to relate the particle zeta potentials with the measured electrophoretic mobility of single particles ${ }^{60}$.

We are using a related approach, in which we are considering the effects of elecroosmosis and convection (through an applied pressure) in addition to electrophoresis when calculating the zeta potential of single particles. Samples of particles with a wide spectrum of zeta potentials, potentially reaching from positive to negative values and/or very dilute suspensions, may require the application of an external pressure in order to capture the whole spectrum of particle zeta potentials. Also, without any net pressure, most neutral particles might not translocate the pore and hence are not measured, skewing the results. 
Average velocities and electric fields at multiple points through the sensing zone (as opposed to only at the end of the sensing zone, see figure 1b) will help to reduce errors that result from rogue events such as instantaneous background noise. In other words, multi point analysis serves as a sort of quality control of the acquired zeta potentials. The calibration of the pore is based on measuring the linear dependence of $1 / T_{x}$ vs voltage, $V$, using standard carboxylated polystyrene particles with a known average zeta potential (figure 1c). The calibration process is summarised in supplementary info (equations A.1-A.6). From this the electrokinetic particle velocities of sample, $\left(v_{x}^{i}\right)_{\text {el sample }}$, and calibration, $\left(v_{x}\right)_{\text {el cal }}$, are related with their zeta potentials, $\xi_{x \text { net sample }}^{i}$ and $\xi_{\text {net cal }}$ (equation 1), assuming a linear relationship between velocity (mobility) and zeta potential as given in the Smoluchowski approximation ${ }^{53,57}$.

$\frac{\left(v_{x}^{i}\right)_{e l ~ S a m p l e}}{\left(v_{x}\right)_{\text {el Cal }}}=\frac{\xi_{x \text { net Sample }}^{i}}{\xi_{\text {net Cal }}}$

The net zeta potentials for both sample and calibration particles are the differences in the respective particle zeta potentials and the membrane zeta potential, $\xi_{m}$ (equation 2).

$\xi_{\text {pample }}=\xi_{\text {net Sample }}+\xi_{m}$

The zeta potential of each sample particle $i$, $\xi_{\text {sample }}^{\mathrm{i}}$, is given by averaging respective zeta potential values, calculated at various locations within the pore (equation 3 ), with $l_{x}$ being the position within the pore reached after time, $t=T_{x}$. Please note that $I_{x}$ is set to equal 0 right at the narrow pore entrance of the conical pore, where the signal magnitude reaches its maximum, as shown in figure 1b. Zeta potentials are evaluated by taking the average at several discrete points, $I_{x}$.

$\xi_{\text {Sample }}^{\mathrm{i}}=\frac{\sum_{\mathrm{x}} \xi_{\mathrm{x} \text { Sample }}^{\mathrm{i}}}{\Sigma_{\mathrm{x}}}=\frac{\sum_{\mathrm{x}}\left(\mathrm{v}_{\mathrm{x} \text { Sample }}^{\mathrm{i}}-\mathrm{v}_{\mathrm{x} \text { Cal }}^{\mathrm{P}} \times P\right) /\left(\mathrm{v}_{\mathrm{x} \text { Cal }}^{\mathrm{V}} \times V\right)}{\Sigma_{\mathrm{x}}} \times \xi_{\text {net Cal }}+\xi_{\mathrm{m}}$

$v_{\mathrm{x} \text { Sample }}^{\mathrm{i}}$ is the sum of the time averaged electrokinetic (electroosmotic and electrophoretic) and convection velocity components of sample particulates at position $I_{x}$ within the pore (equation 4 ).

$v_{\mathrm{x} \text { Sample }}^{\mathrm{i}}=\frac{\mathrm{l}_{\mathrm{x}}}{\mathrm{T}_{\mathrm{x}}^{\mathrm{i}}}=\frac{\int_{0}^{\mathrm{T}_{\mathrm{x}}^{\mathrm{i}}} \mathrm{v}^{\mathrm{i}}(\mathrm{t}) \mathrm{dt}}{\mathrm{T}_{\mathrm{x}}^{\mathrm{i}}}$ 
$\mathrm{v}_{\mathrm{x}}^{\mathrm{V}}{ }_{\text {Cal }}, \mathrm{v}_{\mathrm{x}_{\text {Cal }}}^{\mathrm{P}}, P$, and $V$ are electrokinetic velocity per unit voltage, convective velocity per unit pressure, applied pressure and voltage for the sample runs respectively. The electrokinetic velocity per unit voltage is equivalent to the electrokinetic mobility, which is the sum of electroosmotic and electrophoretic mobility. $v_{\mathrm{x}_{\text {Cal }}}^{\mathrm{V}}$ and $\mathrm{v}_{\mathrm{x}_{\text {Cal }}}^{\mathrm{P}}$ are calculated by averaging typically over more than 500 calibration particles. $\xi_{\text {net cal }}$ and $\xi_{\mathrm{m}}$ are the zeta potentials of polystyrene standard particles and the membrane respectively. The zeta potentials of polystyrene standards and the thermoplastic polyurethane membrane were measured using PALS and streaming potential techniques, to be $-20 \mathrm{mV}$ and $-11 \mathrm{mV}$ respectively (see supplementary info).

\subsection{Zeta potential of DNA Modified Particles}

To test our method on DNA modified particles we first performed a series of measurements increasing the concentration of SsDNA (25 bases in length) on the streptavidin coated particle surfaces. The concentration of DNA, termed here CP, was increased from $10-210 \mathrm{nM}$, whilst the particle concentration remained constant. At DNA concentrations over $188 \mathrm{nM}$ the theoretical binding capacity of the streptavidin particles (as given by the supplier) has been reached (see supplementary figures A.2 and A.3 for the size and charge distributions of the Streptavidin coated particles without DNA). At the highest concentration of DNA added to the particles there is $\sim 12648$ pieces of DNA/ particle, if all of these are attached to the surface of the particles it would equate to 1 DNA molecules every 2 $\mathrm{nm}$ across the particle surface. At lower concentrations of the DNA this ratio changes to $602 /$ bead at $10 \mathrm{nM}$, and 4517/ bead for $75 \mathrm{nM}$. Figure 2 is an example of size and zeta potential data that can be captured simultaneously in a single TRPS measurement. The blue and red bars/data points show the data at the lowest and middle concentration of $\mathrm{CP}$ ( $10 \mathrm{nM}$ and $47 \mathrm{nM}$ respectively) and the green bars/data points show results from the highest concentration of CP measured (210 nM). Please note that each data point in figure 2 represents a single particle. 


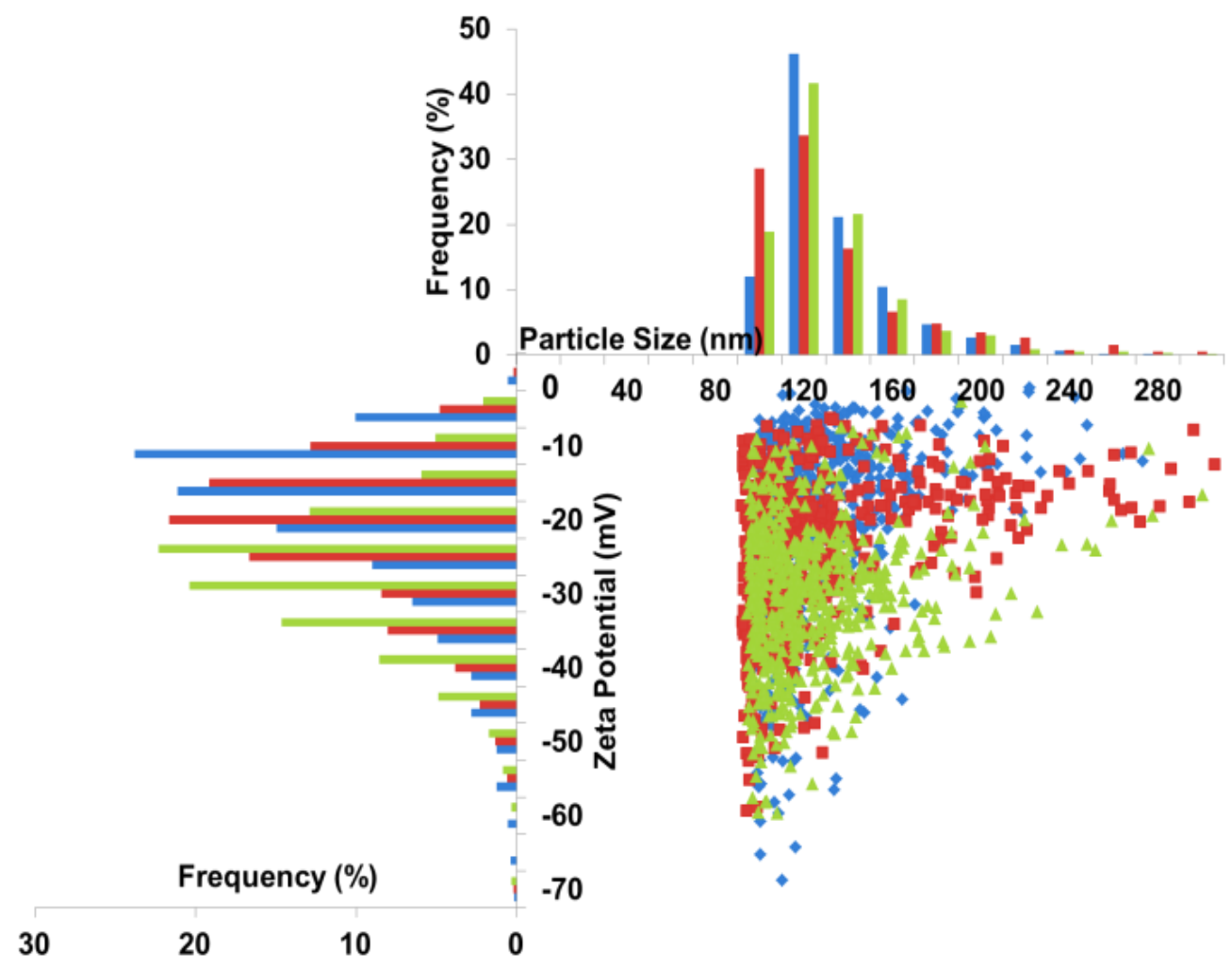

Figure 2 - Size and zeta potential data captured simultaneously from a single TRPS measurement. The blue bars/data points are results of a sample containing $10 \mathrm{nM}$ CP (570 particles measured), the red bars/data points are of a sample containing 47 $n M$ (524 particles measured) and the green bars/data points are of a sample containing 210 nM CP DNA (576 particles measured).

From this, it can be found that although there are no significant size changes observed between the samples, there are significant changes observed in the zeta potential. The inferred zeta potentials from the measured velocities of the samples at varying $\mathrm{CP}$ concentration are shown in figure $3 \mathrm{a}$ and $3 \mathrm{~b}$ respectively. The particle velocities are determined from $1 / \mathrm{T}_{0.50}$ (see supplementary material), which is an estimate of the average particle speeds. The measured zeta potential in figure $3 a$ shows that as the DNA concentration is increased, the larger the absolute zeta potential, and follows the expected trend based on the measured particle velocities; similar data for repeat experiments are given in figure A.2a. This is attributed to each phosphate group contributing to a negative point charge, thereby increasing the charge density of the particle surface, as described by Graham's equation. Surface charge densities were calculated using Graham's equation ${ }^{56,61}$. With mean zeta potentials not exceeding an absolute value of $40 \mathrm{mV}$ the respective absolute surface charge densities lie below $0.035 \mathrm{C} / \mathrm{m}^{2}$, and acknowledge that whilst counter ion condensation may play a role under these situations it is beyond the scope of the 
study to describe in detail. Figure $3 \mathrm{c}$ shows a series of histograms of frequency versus measured zeta potential for each concentration of DNA. The distribution at low concentrations of DNA can be characterised as narrow with a long skewed tail, which may impart be due to the particles themselves not having a uniform coating of streptavidin. As the concentration of DNA is increased the distribution changes, with the median skew value going from $-0.66,-0.36$ and $-0.51-0.36$ for 10,95 and 210 $\mathrm{nM}$ respectively. It should be noted here that the skewed histogram data in the figure may be an inherent property of the particles themselves not having a uniform coating of the streptavidin protein coating, as shown in figure A2. The charge histograms for all of CP concentrations studied are shown in figure A.4 and 5. Particle-by-particle measurements provide more detailed analysis of a sample solution. Charge distribution histograms are used to represent the spread of data amongst a given sample population. The zeta potential of the sample can then be analysed in more depth.

a)

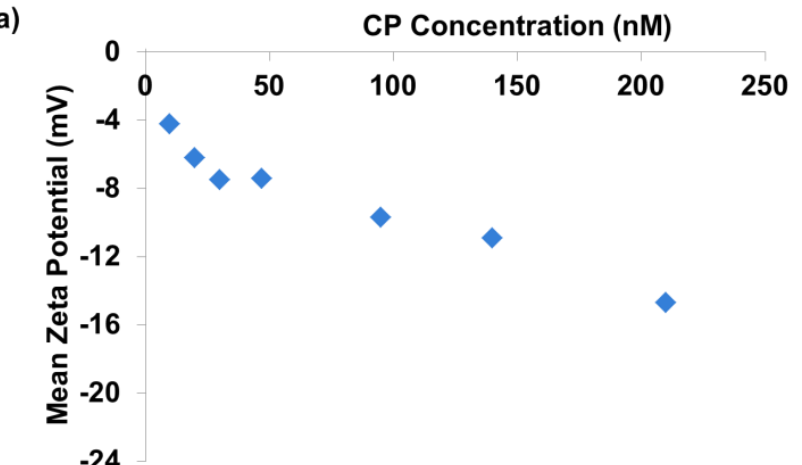

c) $\uparrow$

$10 \mathrm{nM}$

$47 \mathrm{nM}$ b)

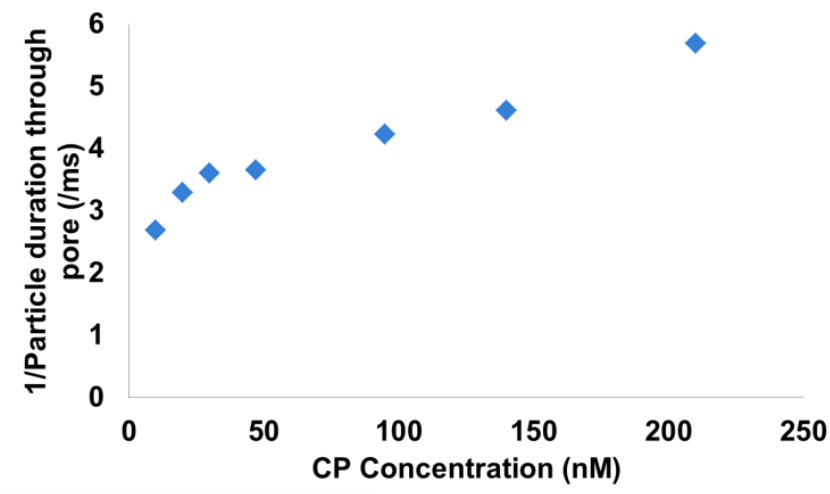

$95 \mathrm{nM}$
$210 \mathrm{nM}$

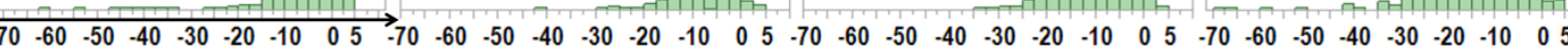

Zeta Potential (mV)

Figure 3 - a) Mean zeta potential vs capture probe concentration. b) $1 / T_{0.50}$, see figure $1 \mathrm{~b}$, estimating average particle speed vs capture probe concentration. c) Charge distributions amongst the sample population shown in 'a)' increasing in DNA concentration from left to right. 537, 605, 585, and 588 particles were measured for the samples containing 10,47, 95, and $210 \mathrm{nM}$ DNA respectively.

A similar relationship between the length of the ssDNA and measured zeta potential should also exist, that is as the length of the DNA increases, the zeta potential is also predicted to increase. Steinbock et al. have previously investigated the effect of long double stranded DNA strands ( 4 and 6 kilo base pairs) hybridised 
to colloids using microparticles and a microcapillary base Coulter counter system. They found that DNA coated microparticles displayed a much smaller change in conductance values due to the additional charge in the system ${ }^{40}$. To investigate the sensitivity of TRPS in its ability to measure changes in zeta potential, we are focusing on much smaller strands of single-stranded DNA. The ssDNA oligonucleotides in this study were $10,25,36$ and 50 bases in length equivalent to $7.0,17.5,25.2$ and $35 \mathrm{~nm}$ in length respectively if the ssDNA is fully extended ${ }^{62,63}$.

a)

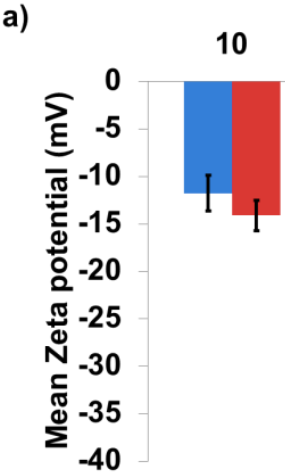

c)

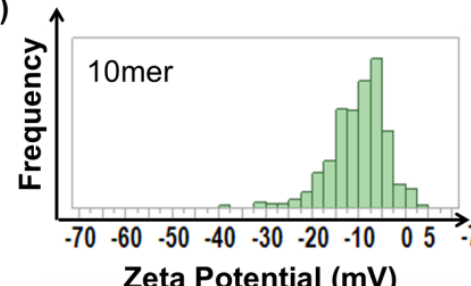

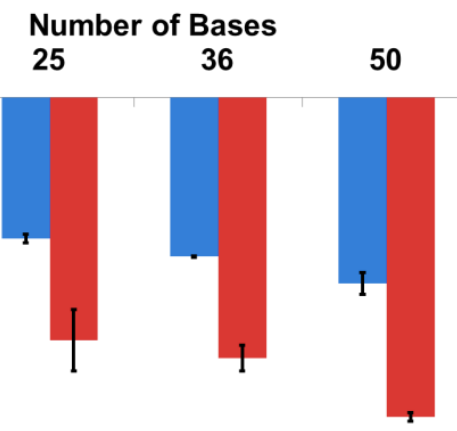

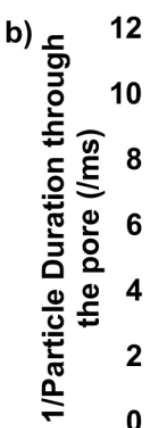

$25 \mathrm{mer}$

Figure 4 - a) Mean zeta potential vs capture probe base length. Blue bars are representative of a $75 \mathrm{nM}$ DNA concentration and red bars represent a $210 \mathrm{nM}$ DNA concentration. b) $1 / T_{0.50}$, see figure $1 b$, estimating average particle speed vs capture probe base length. c) The charge distribution of varied DNA base length using 210 nM DNA. 676, 1001, 996, and 693 particles were measured for the 10, 25, 36, and $50 \mathrm{mer}$ respectively. Error bars represent st.dev where $n=2$.

Figure $4 a$ shows how the zeta potential increases when the length of the DNA is increased alongside the relative particle velocity displayed in figure $4 \mathrm{~b}$. In this example (red bars), the concentration of DNA added to the particles is in excess of the binding capacity. Figure $4 a$, blue bars, illustrate the same effect, i.e. as the length of the DNA increases so does the measured zeta potential. However in this experiment, the DNA is at $75 \mathrm{nM}$, which is lower than the theoretical binding capacity of the particles. At this lower concentration the DNA is much more flexible and can exist in its condensed mushroom form ${ }^{64}$. Figure $4 c$ shows the charge distribution histograms for the densely packed DNA particles (distributions for the 75nM DNA are given in figure A.6, and distributions from multiple runs are presented in figure A.7.). 
The width of the distribution increases as the length of the DNA increases (D90/10 goes from 2.87 to 5.3 for 10 and 50 base lengths respectively), we attribute this observation to the steric hindrance of the longer strands, preventing a higher packing density of the DNA around the particles due to their radius of gyration, effectively blocking the binding of the DNA onto the surface. Its also interesting to note that the median skewness values from -042 to $-0.41,-0.12$ and 0.45 for 10,25 , 35 and 50 bases respectively. The particle charge distributions for the lower DNA concentration $(75 \mathrm{nM})$ and comparative datasets for the higher concentration (210 $\mathrm{nM}$ ) illustrating the reproducibility of the data are given in supplementary figures A.6 and A.7.

\subsection{Detecting Target DNA Hybridisation}

It was then investigated if the technique could discriminate between ssDNA and dsDNA. Initially the capture probe length was kept constant at 25 bases in length, and was always added in excess of the binding capacity. Any unbound capture probe in solution was removed before the target probe was incubated with the particles. As can be seen in figure 5ai and ii, the formation of dsDNA can be measured by an increase in zeta potential for assay hybridisation times of 16 hours (green triangle) and for hybridisation times as short as 30 minutes (red squares).

The change in structure from ssDNA to dsDNA is a 50 -fold increase in persistence length ${ }^{30,31}$, and this will result in the hydrodynamic radius of the particle upon forming dsDNA to increase. Two competing factors then affect the surface chare density. The first is the elongation of the DNA upon forming the dsDNA structure which has the effect of spacing out the charged phosphate groups away from the particles surface, resulting in a decrease in charge density. However this is countered by the addition of a second strand of DNA doubling the number of point charges resulting in a net increase in electrophoretic mobility in solution, and thus resulting in increased velocities and larger zeta potential values.

This is similar to the work done by Booth et al. demonstrating the detection of target-probe DNA hybridisation and successfully discriminating between 'probe' and 'target-probe' hybridised particles using TRPS ${ }^{65}$. However, in these previous examples the experiments utilised a $23 \mathrm{mer}$ capture probe and $50 \mathrm{mer}$ target, as such the captured DNA extended out into solution and was predominantly ssDNA. Here 
we were curious as to the ability of the technique to discriminate between SSDNA and dsDNA, as well as overhanging DNA. We investigated a range of DNA targets binding to various positions of the capture probe, to determine the sensitivity and reliability of a zeta potential measurement for the detection of varied DNA hybridisation. We termed these target probes as CDNA (fully complementary), MidT (binds to the middle of the CP), EndT (binds to the end of the CP) and OverT (binds to the end 10 bases of the CP and overhangs into solution by 15 bases). The results for these measured zeta potential values are plotted in figure 5aiii-v. As we add target DNA in each of the hybridisation experiments to form dsDNA, be it at the middle or end of the $\mathrm{CP}$, there is a larger zeta potential recorded. The magnitude of change in zeta potential is always greatest with the longer hybridisation times. Of interest is that fact the overhanging DNA sequences (OverT) gives the largest negative zeta potential of all the samples despite being the longest length. Increasing the length of the DNA could have slowed the speed at which the particles traverse the pore due to additional drag effects and lowered the recorded zeta potential. However, the result indicates that the increase in charge due to the additional 30 based has a more dominant effect on particle translocation times. One suggestion for this observation could be down to the ssDNA having a lower persistence length. The overhanging ssDNA may coil/ fold back towards the particle. This folding in effect increased the surface charge density around the particle increasing the electrophoretic velocity through the creation of a "hairy layer mechanism,66. The ssDNA within the overhanging DNA is also further from the particles surface than the DNA in any other experiment. Given the curvature of the particle the distance between each DNA molecule will increase, and this room to fold back may explain the enhanced effect over MidT and EndT experiments. 


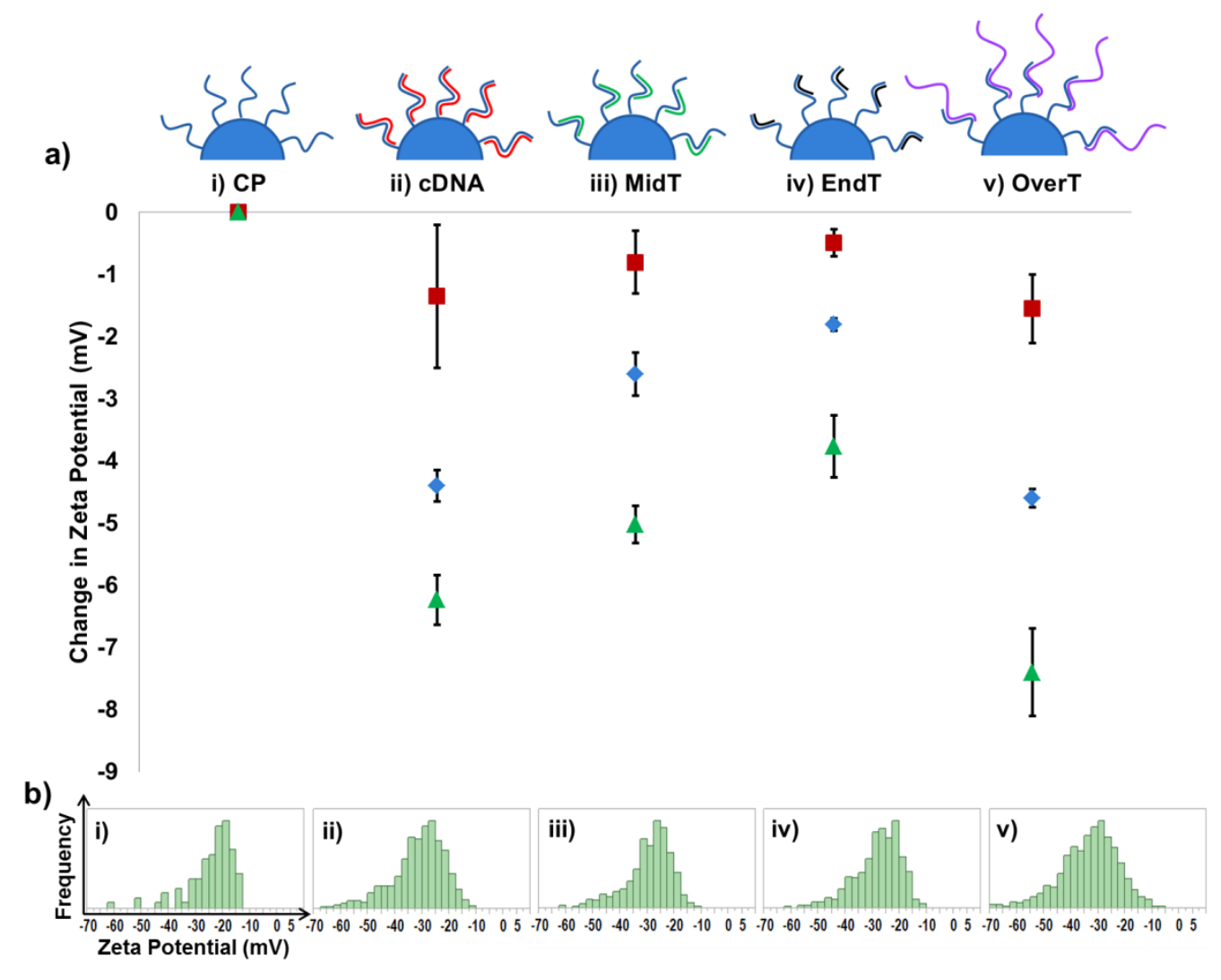

Figure 5 - a) Relative change in mean zeta potential $(m V)$ from $D N A$ capture probe $(C P, 250 \mathrm{nM})$ to when a variety of targets are hybridised for 30 minutes (red squares) and hybridised for 16 hours (green triangles). The relative change in zeta potential was also investigated for a lower concentration (75 nM) and 30 minute hybridisation time (blue diamonds). b) Charge distributions for each target at $250 \mathrm{nM} \mathrm{CP}$ concentrations and a hybridisation time of 16 hours. 500, 990, 592, 707, and 964 particles were measured for samples i)-v) respectively with median skewness values of -073, $-0.59,-0.41,-0.49,-0.31$. Error bars represent st.dev where $n=3$.

A surprising aspect of the data was the ability to distinguish between dsDNA formed at the end or middle of the capture probe. The target DNA that bound to the end of the CP (EndT), recorded a smaller zeta potential than a same sized target that was hybridised to the middle of the CP (MidT). We attribute this to the effects of persistence lengths and the location of the dsDNA in the capture probe. The MidT dsDNA leaves a section of ssDNA exposed to the solution. This is more flexible and we hypothesise that when the dsDNA is in the middle of the DNA, the single stranded end section coils/ folds back to increase the charge density around the particle ${ }^{66}$, thus creating a larger zeta potential. In the case where the dsDNA is at the end of the sequence, the ability of the DNA to fold back on its self is restricted and forms a more rigid elongated oligomer across the entire length of the DNA, moving the charge away from the surface and lowering the surface charge density. 
The observation that MidT produces a larger shift in zeta potential could influence the design of future assays on TRPS systems. The change in distribution shape indicates a difference in the DNA hybridisation or DNA target itself. Figure 5b shows the change in charge histogram shape, dependent on the target DNA hybridised to the CP. The ability to monitor any of these discrete differences is an insightful prospect for future colloid and nucleotide research.

As well as designing the location to capture target DNA, there is also an interesting observation on the effect of DNA density on hybridisation kinetics. Previous studies have illustrated that the kinetics of target DNA capture is influenced by DNA probe density at a surface ${ }^{67-70}$. At high DNA probe densities, the SsDNA forms a dense packed polymer brush ${ }^{56}$, the DNA forms a rigid polymer coating who's thickness is equal to the length of the extended DNA sequence, $H^{72}$. The effects on packing density then determine the electrostatic potential, the position of the shear plane and the kinetics of target DNA hybridisation. The significance of the zeta potential at polyelectrolyte layers becomes more complicated, and when the debye length, $\kappa^{-1}$, is sufficiently lower than the polyelectrolyte layer thickness, $H$, i.e. $\kappa^{-}$ $1 / H<<1$, the measured zeta potential may no longer reflect the stern potential, as the plane of shear is shifted to distances further away from the particles surface ${ }^{73}$. Therefore at polyelectrolyte surfaces, the term zeta potential in effect loses its original meaning. As the density of the DNA packing decreases the plane of shear may enter the DNA layer ${ }^{73}$.

Figure 6 shows the effect of hybridising an excess of target cDNA to different packing densities of $\mathrm{CP}$. At a low capture probe concentration and a target hybridisation time of 30 minutes (shown by figure 6a), the charge distributions were much narrower in shape with less of a tailing effect observed. In agreement with previous studies ${ }^{74}$, when the DNA capture probe concentration is lower, there is a faster rate of reaction, resulting in a much narrower charge distribution histogram. At high capture probe concentrations, it is difficult to observe an increase in zeta potential for small hybridisation reaction times, thus for quick assay times, low packing densities of CP produce better results. 


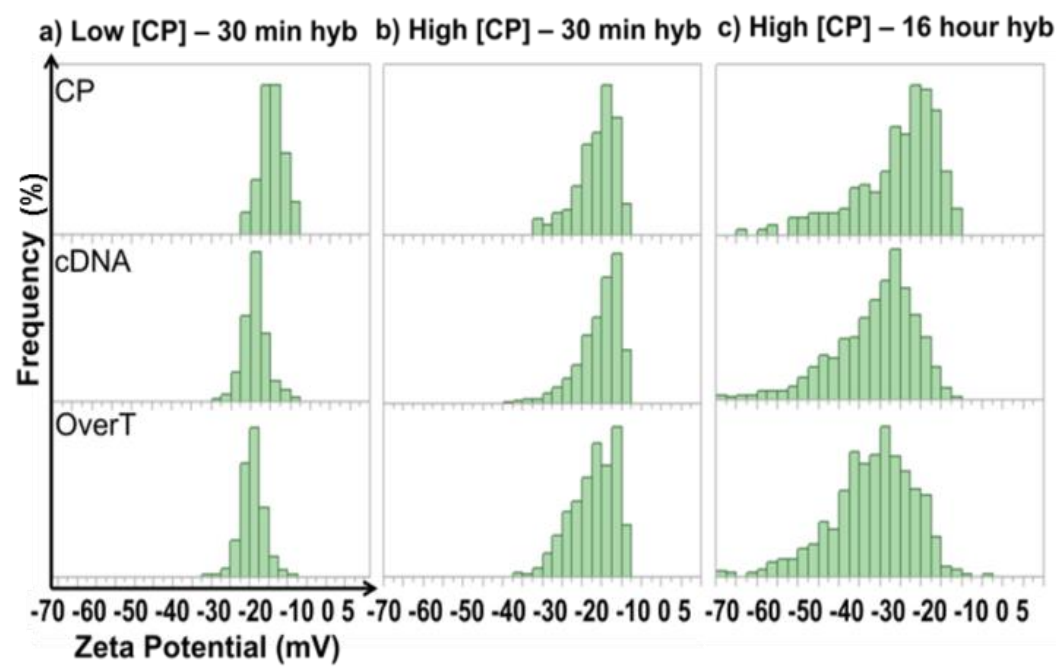

Figure 6 - The charge distributions for a sample population at; a) low concentration of capture probe $(75 \mathrm{nM})+30$ minute hybridisation of varied targets in excess, (987, 998, and 996 particles were measured for $C P, C D N A$, and OverT respectively) b) high concentration of capture probe $(250 \mathrm{nM})+30$ minute hybridisation of varied targets in excess, (502, 997, and 661 particles were measured for CP, cDNA and OverT respectively) and c) high concentration of capture probe (250 nM) + 16 hour hybridisation of varied targets in excess $(512,916$, and 944 particles were measured for $C P, c D N A$ and OverT respectively).

Figure $6 \mathrm{~b}$ and $6 \mathrm{c}$ both display results observed at a high CP concentrations and show the charge distribution widening as the target hybridisation time increases from 30 minutes to 16 hours. This is due to the increase in amount of time the target DNA has to reach the required orientation to achieve successful complementary DNA binding. The more time there is for this to happen, the higher the proportion of target DNA that can successfully bind to the capture probe resulting in a larger amount of dsDNA present on the particles. This increases the particle velocity through the nanopore, thus resulting in a larger absolute zeta potential.

A more Gaussian charge distribution was seen for particles analysed in figure $6 \mathrm{a}$ and $6 c$ than in $6 b$, skewness values of $0.1,-0.41$ and -0.37 respectively. We attribute this to an increase in ability to form dsDNA, and then detect its presence on a particle. For example with a hybridisation time of 30 minutes and a lower capture probe concentration (6a), the presence of dsDNA is easily detected. This may be due to two factors, firstly there being less steric hindrance for the target DNA to approach the particle allowing the rate of dsDNA formation to be increased, and 
secondly the resolution of the technique to measure the incremental additional of dsDNA against a particle of lower charge, compared to a high density ssDNA covered particle in $6 \mathrm{~b}$. Increasing the target hybridisation time to 16 hours (figure $6 \mathrm{c}$ ) using the high concentrations of capture probe allows the target to have more time hybridise and thus more dsDNA is present on the surface.

\section{Conclusions}

We have demonstrated that TRPS can successfully detect and characterise both unmodified and DNA-modified particles in a single, real-time measurement. Charge distributions, rather than a single mean zeta potential value allow for more information to be extracted from a sample dataset using a particle-by-particle perspective. DNA-based surface modifications to a nanoparticle affect the behaviour of the nanoparticles in an electrolyte solution and their mobility through a nanopore; and by optimising the hybridisation time and DNA packing density on a surface, we measure the successful capture of target DNA after just 30 minutes incubation time. Successful analyte capture after such short incubation times is advantageous and shows great potential for medical applications, such as point of care assays, for example.

\section{Acknowledgments}

The authors thank Izon Scientific Ltd for their support and the Centre for Analytical Science at Loughborough University. The work was supported by the European Commission for Research (PCIG11-GA-2012-321836 Nano4Bio), Loughborough University Chemistry Department (Start-up fund) and by the National Institute for Health Research (NIHR) Diet, Lifestyle \& Physical Activity Biomedical Research Unit based at University Hospitals of Leicester and Loughborough University. The views expressed are those of the authors and not necessarily those of the NHS, the NIHR or the Department of Health. E. L. C. J. B. is supported by Izon Science Ltd.

\section{Appendix A - Supplementary Material}

Supplementary data associated with this article can be found in the online version at: 


\section{Conflicts of Interest Disclosure}

The authors declare no competing financial interest.

\section{References}

1. Niemeyer, C. M. \& Blohm, D. DNA Microarrays. Angew. Chemie 38, 2865-2869 (1999).

2. Gresham, D., Dunham, M. J. \& Botstein, D. Comparing whole genomes using DNA microarrays. Nat Rev Genet 9, 291-302 (2008).

3. Bulyk, M. L. Protein Binding Microarrays for the Characterization of Protein-DNA Interactions. Adv. Biochem. Eng. Biotechnol. 104, 65-85 (2007).

4. Platt, M., Rowe, W., Knowles, J., Day, P. J. \& Kell, D. B. Analysis of aptamer sequence activity relationships. Integr. Biol. 1, 116-122 (2009).

5. Billinge, E. R. \& Platt, M. Aptamer based dispersion assay using tunable resistive pulse sensing (TRPS). Anal. Methods 7, 8534-8538 (2015).

6. Billinge, E. R., Broom, M. \& Platt, M. Monitoring Aptamer-Protein Interactions Using Tunable Resistive Pulse Sensing. Anal. Chem. 86, 1030-1037 (2013).

7. Gold, L. \& al., et. Aptamer-Based Multiplexed Proteomic Technology for Biomarker Discovery. PLoS One 5, e15004 (2010).

8. Park, S.-J., Taton, T. A. \& Mirkin, C. A. Array-Based Electrical Detection of DNA with Nanoparticle Probes. Science (80-. ). 295, 1503-1506 (2002).

9. Billinge, E. R. \& Platt, M. Multiplexed, label-free detection of biomarkers using aptamers and Tunable Resistive Pulse Sensing (AptaTRPS). Biosens. Bioelectron. 68, 741-748 (2015).

10. Ruiz-Hernández, E., Baeza, A. \& Vallet-Regí, M. Smart Drug Delivery through DNA/Magnetic Nanoparticle Gates. ACS Nano 5, 1259-1266 (2011).

11. Alexander, C. M., Maye, M. M. \& Dabrowiak, J. C. DNA-capped nanoparticles designed for doxorubicin drug delivery. Chem. Commun. 47, 3418-3420 (2011).

12. Li, J., Fan, C., Pei, H., Shi, J. \& Huang, Q. Smart Drug Delivery Nanocarriers with SelfAssembled DNA Nanostructures. Adv. Mater. 25, 4386-4396 (2013).

13. Rosi, N. L. \& Mirkin, C. A. Nanostructures in Biodiagnostics. Chem. Rev. 105, 1547-1562 (2005).

14. Llandro, J., Palfreyman, J., Ionescu, A. \& Barnes, C. Magnetic biosensor technologies for medical applications: a review. Med. Biol. Eng. Comput. 48, 977-998 (2010).

15. Tokel, O., Inci, F. \& Demirci, U. Advances in Plasmonic Technologies for Point of Care Applications. Chem. Rev. (2014). doi:10.1021/cr4000623

16. Michalet, X. et al. Quantum Dots for Live Cells, in Vivo Imaging, and Diagnostics. Science (80. ). 307, 538-544 (2005). 
17. Salata, O. V. Applications of nanoparticles in biology and medicine. J. Nanobiotechnology 2, 3 (2004).

18. Sun, J., Xianyu, Y. \& Jiang, X. Point-of-care biochemical assays using gold nanoparticleimplemented microfluidics. Chem. Soc. Rev. 43, 6239-6253 (2014).

19. Thaxton, C. S. et al. Nanoparticle-based bio-barcode assay redefines 'undetectable' PSA and biochemical recurrence after radical prostatectomy. Proc. Natl. Acad. Sci. U. S. A. 106, 18437-18442 (2009).

20. McCarthy, J. R. \& Weissleder, R. Multifunctional magnetic nanoparticles for targeted imaging and therapy. Adv. Drug Deliv. Rev. 60, 1241-1251 (2008).

21. Kim, H., Achermann, M., Balet, L. P., Hollingsworth, J. A. \& Klimov, V. I. Synthesis and characterization of $\mathrm{Co} / \mathrm{CdSe}$ core/shell nanocomposites: Bifunctional magnetic-optical nanocrystals. J. Am. Chem. Soc. 127, 544-546 (2005).

22. Pamme, N. \& Wilhelm, C. Continuous sorting of magnetic cells via on-chip free-flow magnetophoresis. Lab Chip 6, 974-980 (2006).

23. Whitaker, J. R. et al. Antibody-based enrichment of peptides on magnetic beads for massspectrometry-based quantification of serum biomarkers. Anal. Biochem 1, 44-54 (2007).

24. Pamme, N. Continuous flow separations in microfluidic devices. Lab Chip 7, 1644-1659 (2007).

25. Kojima, T. et al. PCR amplification from single DNA molecules on magnetic beads in emulsion: application for high-throughput screening of transcription factor targets. Nucleic Acids Res. 33, e150 (2005).

26. Park, S., Sinha, N. \& Hamad-Schifferli, K. Effective Size and Zeta Potential of Nanorods by Ferguson Analysis. Langmuir 26, 13071-13075 (2010).

27. Norde, W. Colloids and Interfaces in Life Sciences. Materials Today 6, (CRC Press, Taylor and Francis Group, 2011).

28. Clogston, J. \& Patri, A. in Characterization of Nanoparticles Intended for Drug Delivery SE - 6 (ed. McNeil, S. E.) 697, 63-70 (Humana Press, 2011).

29. Park, S. \& Hamad-Schifferli, K. Evaluation of Hydrodynamic Size and Zeta-Potential of Surface-Modified Au Nanoparticle-DNA Conjugates via Ferguson Analysis. J. Phys. Chem. C 112, 7611-7616 (2008).

30. Tinland, B., Pluen, A., Sturm, J. \& Weill, G. Persistence Length of Single-Stranded DNA. Macromolecules 30, 5763-5765 (1997).

31. Bustamante, C., Bryant, Z. \& Smith, S. B. Ten years of tension: single-molecule DNA mechanics. Nature 421, 423-427 (2003).

32. De las Nieves, F. J., Daniels, E. S. \& El-Aasser, M. S. Electrokinetic characterization of highly sulfonated polystyrene model colloids. Colloids and Surfaces 60, 107-126 (1991).

33. Mullen, D. G. et al. A Quantitative Assessment of Nanoparticle Ligand Distributions: Implications for Targeted Drug and Imaging Delivery in Dendrimer Conjugates. ACS Nano 4, 657-670 (2010). 
34. Mullen, D. G. \& Banaszak Holl, M. M. Heterogeneous Ligand-Nanoparticle Distributions: A Major Obstacle to Scientific Understanding and Commercial Translation. Acc. Chem. Res. 44, 1135-1145 (2011).

35. Uddayasankar, U., Shergill, R. T. \& Krull, U. J. Evaluation of Nanoparticle-Ligand Distributions To Determine Nanoparticle Concentration. Anal. Chem. 87, 1297-1305 (2015).

36. Jin, R., Wu, G., Li, Z., Mirkin, C. A. \& Schatz, G. C. What Controls the Melting Properties of DNA-Linked Gold Nanoparticle Assemblies? J. Am. Chem. Soc. 125, 1643-1654 (2003).

37. Geerts, N. \& Eiser, E. DNA-functionalized colloids: Physical properties and applications. Soft Matter 6, 4647 (2010).

38. Jones, M. R., Macfarlane, R. J., Prigodich, A. E., Patel, P. C. \& Mirkin, C. A. Nanoparticle Shape Anisotropy Dictates the Collective Behavior of Surface-Bound Ligands. J. Am. Chem. Soc. 133, 18865-18869 (2011).

39. Rant, U. et al. Excessive counterion condensation on immobilized ssDNA in solutions of high ionic strength. Biophys. J. 85, 3858-64 (2003).

40. Steinbock, L. J., Stober, G. \& Keyser, U. F. Sensing DNA-coatings of microparticles using micropipettes. Biosens. Bioelectron. 24, 2423-7 (2009).

41. Gagnon, Z., Senapati, S. \& Chang, H.-C. Optimized DNA hybridization detection on nanocolloidal particles by dielectrophoresis. Electrophoresis 31, 666-671 (2010).

42. Russell, A. J., Del Bonis-O'Donnell, J. T., Wynne, T. M., Napoli, M. T. \& Pennathur, S. Separation behavior of short single- and double-stranded DNA in 1 micron and $100 \mathrm{~nm}$ glass channels. Electrophoresis 35, 412-418 (2014).

43. Devasenathipathy, S., Santiago, J. G. \& Takehara, K. Particle Tracking Techniques for Electrokinetic Microchannel Flows. Anal. Chem. 74, 3704-3713 (2002).

44. Kozak, D. et al. Simultaneous size and $\zeta$-potential measurements of individual nanoparticles in dispersion using size-tunable pore sensors. ACS Nano 6, 6990-6997 (2012).

45. Kozak, D., Anderson, W., Vogel, R. \& Trau, M. Advances in Resistive Pulse Sensors: Devices bridging the void between molecular and microscopic detection. Nano Today 6, 531-545 (2011).

46. Roberts, G. S. et al. Tunable nano/micropores for particle detection and discrimination: scanning ion occlusion spectroscopy. Small 6, 2653-8 (2010).

47. Roberts, G. S. et al. Tunable pores for measuring concentrations of synthetic and biological nanoparticle dispersions. Biosens. Bioelectron. 31, 17-25 (2012).

48. Sowerby, S. J., Broom, M. F. \& Petersen, G. B. Dynamically resizable nanometre-scale apertures for molecular sensing. Sensors Actuators B Chem. 123, 325-330 (2007).

49. Vogel, R. et al. Quantitative sizing of nano/microparticles with a tunable elastomeric pore sensor. Anal. Chem. 83, 3499-3506 (2011).

50. Willmott, G. R. et al. Use of tunable nanopore blockade rates to investigate colloidal dispersions. J. Phys. Condens. Matter 22, 454116 (2010). 
51. Blundell, E. L. C. J., Mayne, L. J., Billinge, E. R. \& Platt, M. Emergence of tunable resistive pulse sensing as a biosensor. Anal. Methods Advance Ar, 1-12 (2015).

52. Willmott, G. R., Platt, M. \& Lee, G. U. Resistive pulse sensing of magnetic beads and supraparticle structures using tunable pores. Biomicrofluidics 6, 14103-1410315 (2012).

53. Kozak, D., Anderson, W., Vogel, R. \& Chen, S. Simultaneous size and $\zeta$-potential measurements of individual nanoparticles in dispersion using size-tunable pore sensors. ACS ... 6, 6990-6997 (2012).

54. Vogel, R., Anderson, W., Eldridge, J., Glossop, B. \& Willmott, G. A variable pressure method for characterizing nanoparticle surface charge using pore sensors. Anal. Chem. 84, 31253131 (2012).

55. Eldridge, J. a., Willmott, G. R., Anderson, W. \& Vogel, R. Nanoparticle $\zeta$-potential measurements using tunable resistive pulse sensing with variable pressure. J. Colloid Interface Sci. 429, 45-52 (2014).

56. Arjmandi, N., Van Roy, W., Lagae, L. \& Borghs, G. Measuring the electric charge and zeta potential of nanometer-sized objects using pyramidal-shaped nanopores. Anal. Chem. 84, 8490-8496 (2012).

57. Hunter, R. J. Zeta Potential in Colloid Science: Principles and Applications. (Academic Press: London, 1981).

58. Overbeek, J. T. G. \& Wiersema, P. H. in Electrophoresis: Theory, Methods and Applications (ed. Bier, M.) 1-52 (Academic Press: New York, 1967).

59. Willmott, G. R. \& Parry, B. E. T. Resistive pulse asymmetry for nanospheres passing through tunable submicron pores. J. Appl. Phys. 109, (2011).

60. Henry, D. C. The Cataphoresis of Suspended Particles, Part 1: The equation of cataphoresis. (Proc. Roy. Soc., 1931).

61. Daiguji, H., Yang, P. \& Majumdar, A. Ion Transport in Nanofluidic Circuits. Nano Lett. 4, 47405 (2004).

62. Smith, S. B., Cui, Y. \& Bustamante, C. Overstretching B-DNA: the elastic response of individual double-stranded and single-stranded DNA molecules. Science 271, 795-799 (1996).

63. Record, M. T., Anderson, C. F. \& Lohman, T. M. Thermodynamic analysis of ion effects on the binding and conformational equilibria of proteins and nucleic acids: the roles of ion association or release, screening, and ion effects on water activity. Q. Rev. Biophys. 11, 103-178 (1978).

64. Rao, A. \& Grainger, D. Biophysical properties of nucleic acids at surfaces relevant to microarray performance. Biomater. Sci. 436-471 (2014). doi:10.1039/c3bm60181a

65. Booth, M. A., Vogel, R., Curran, J. M., Harbison, S. \& Travas-Sejdic, J. Detection of targetprobe oligonucleotide hybridization using synthetic nanopore resistive pulse sensing. Biosens. Bioelectron. 45, 136-40 (2013).

66. Hidalgo-Álvarez, R. et al. Electrokinetic properties, colloidal stability and aggregation kinetics of polymer colloids. Adv. Colloid Interface Sci. 67, 1-118 (1996).

67. Southern, E., Mir, K. \& Shchepinov, M. Molecular interactions on microarrays. Nat. Genet. 21, S5-S9 (1999). 
68. Huang, E., Satjapipat, M., Han, S. \& Zhou, F. Surface structure and coverage of an oligonucleotide probe tethered onto a gold substrate and its hybridization efficiency for a polynucleotide target. Langmuir 17, 1215-1224 (2001).

69. Peterson, A., Heaton, R. \& Georgiadis, R. The effect of surface probe density on DNA hybridization. Nucleic Acids Res. 29, 5163-5168 (2001).

70. Randeria, P. S. et al. What Controls the Hybridization Thermodynamics of Spherical Nucleic Acids? J. Am. Chem. Soc. 137, 3486-3489 (2015).

71. Wong, I. Y. \& Melosh, N. A. An Electrostatic Model for DNA Surface Hybridization. Biophys. J. 98, 2954-2963 (2010).

72. Valignat, M.-P., Theodoly, O., Crocker, J. C., Russel, W. B. \& Chaikin, P. M. Reversible selfassembly and directed assembly of DNA-linked micrometer-sized colloids. Proc. Natl. Acad. Sci. U. S. A. 102, 4225-4229 (2005).

73. Shen, G. et al. Charging behavior of single-stranded DNA polyelectrolyte brushes. J. Am. Chem. Soc. 128, 8427-8433 (2006).

74. Halperin, A., Buhot, A. \& Zhulina, E. B. On the hybridization isotherms of DNA microarrays: the Langmuir model and its extensions. Journal of Physics: Condensed Matter 18, S463-S490 (2006). 